\section{GW23-e2117 POSTCONDITIONING EFFECTS OF ORAL NICORANDIL TREATMENT ON PATIENTS WITH ACUTE ANTERIOR MYOCARDIAL INFARCTION}

doi:10.1136/heartjnl-2012-302920l.2

Li Feng, Tong Liu, Li Feng. Zhongshan City, Guangdong Province People's hospital of Zhongshan, Cardiovascular Department

Objectives In recent years, the development of thrombolysis and percutaneous coronary intervention (PCI) have positive impacts on outcome of the patients with acute myocardial infarction (AMI), but the reperfusion injury followed by these treatments per se would lower the benefit. After carrying on a large amount of research, people have found that ischaemic preconditioning can decrease the reperfusion injury and reduce the infarct size, which is a strong endogenous myocardial protective form of reducing reperfusion injury. But it must be operated in a transient time before myocardial ischaemia, which is the bottleneck of its wide clinical application. But recent research has found that ischaemic post conditioning can exert cardio protective effect as well when operated after a short period of myocardial ischaemia, which has become a hotspot of coronary heart disease therapy researches. Since the mitochondria potassium ATP (mito-KATP) channel is at the downstream of signal pathway of preconditioning and post conditioning, the activation of mito-KATP channel can directly induce preconditioning and postconditioning. Nicorandil is a medicine which can act as agonist of mito-KATP channel and has nitrates effect. The pharmacologic preconditioning caused by Nicorandil has been confirmed in animal experiments and clinical tests. By activating mitoKATP channel, Nicorandil can cause pharmacologic post conditioning, theoretically. However, at present there is only a few animal experiments validating the post conditioning property of nicorandil. No further clinical experiments develops this topic. To test whether nicorandil can induce pharmacologic postconditioning or not, and to test whether it can reduce the infarct size of the acute anterior myocardial infarction, which achieve the result of limiting the injury of reperfusion.

Methods According to the criteria of inclusion and exclusion, the final experimental subjects are 43 patients with acute anterior myocardial infarction. All of them were treated in the emergency department of Zhongshan People's Hospital during the period of March 2011 to January 2012. They were randomly divided into two groups. There were 21 patients in group NIC and 22 in group ISDN. Patients in NIC group were taken $10 \mathrm{mg}$ Nicorandil (NIC) orally before PCI procedure and treated with $5 \mathrm{mg}$ Nicorandil three times a day for 7 days. Patients in ISDN group were given
Isosorbide Dinitrate (ISDN) in the same method. By observing the changes of ECG ST-segment before operation and $1 \mathrm{~h}$ after operation, the ST-segment resolution rate was gained; and the blood samples were taken from all patients before operation and $6 \mathrm{~h}$, $12 \mathrm{~h}, 18 \mathrm{~h}, 24 \mathrm{~h}, 48 \mathrm{~h}, 72 \mathrm{~h}$ after operation, which are used to observe the dynamic changes of CKMB. At last, the ECT myocardial perfusion examinations were performed in the patients on the 7 th day after operation, which can reflect the infarct size of the acute anterior myocardial infarction.

Results Compared with the ISDN, nicorandil can considerably reduce the peak value of creatine kinase myocardial band. NIC group $(171.6 \pm 105.7 \mathrm{U} / \mathrm{l})$ vs ISDN group $(246.4 \pm 108.4 \mathrm{U} / \mathrm{l})$, $\mathrm{p}<0.05$. They can also reduce the area under the curve. NIC group $(4309 \pm 2575)$ vs ISDN group $(5854 \pm 2352), p<0.05$. In addition, nicorandil can reduce the proportion of ECT myocardial perfusion defect extent in left ventricle: NIC Group (30.38 $\pm 11.83 \%)$ vs ISDN group $(38.64 \pm 14.59 \%), p<0.05$. And ECT perfusion defect score is: NIC Group $(25.19 \pm 11.96)$ vs ISDN group (33.27 \pm 14.03$)$, $\mathrm{p}<0.05$. Moreover, NIC group had a better ECG ST-segment resolution. There are 18 patients in NIC group and 13 patients in ISDN group having experienced ST-segment completely resolution. The ST-segment resolution rate of the two groups are $85.71 \%$ and $59.09 \%, \mathrm{p}<0.05$, respectively.

Conclusions (1) Before giving reperfusion in the patients with acute anterior myocardial infarction, oral nicorandil treatment within $1 \mathrm{~h}$ can improve ECG ST-segment resolution, reduce the peak value of $\mathrm{CKMB}$ and the area under the curve of $\mathrm{CKMB}$, and decrease the proportion of ECT myocardial perfusion defect extent in left ventricle and ECT perfusion defect score. (2) By activating the mito-KATP channel, nicorandil can induce pharmacologic post conditioning effect, limit the infarct size of the acute anterior myocardial infarction and decrease the reperfusion injury, which eventually form cardio protective effects. Besides, this effect is no related to nitrate effect. 